\title{
Neuronal adaptation, novelty detection and regularity encoding in audition
}

\author{
Manuel S. Malmierca ${ }^{1,2}{ }^{*}$, Maria V. Sanchez-Vives ${ }^{3,4}$, Carles Escera ${ }^{5,6}$ and Alexandra Bendixen ${ }^{7}$ \\ ' Auditory Neurophysiology Unit, Laboratory for the Neurobiology of Hearing, Institute of Neuroscience of Castilla y León, University of Salamanca, Salamanca, Spain \\ ${ }^{2}$ Department of Cell Biology and Pathology, Faculty of Medicine, University of Salamanca, Salamanca, Spain \\ ${ }^{3}$ Institució Catalana de Recerca i Estudis Avançats (ICREA), Barcelona, Spain \\ ${ }^{4}$ Institut de Investigacions Biomèdiques August Pi i Sunyer (IDIBAPS), Barcelona, Spain \\ ${ }^{5}$ Institute for Brain, Cognition and Behavior (IR3C), University of Barcelona, Barcelona, Spain \\ ${ }^{6}$ Cognitive Neuroscience Research Group, Department of Psychiatry and Clinical Psychobiology, University of Barcelona, Barcelona, Spain \\ ${ }^{7}$ Auditory Psychophysiology Lab, Department of Psychology, Cluster of Excellence "Hearing4all," European Medical School, Carl von Ossietzky University of \\ Oldenburg, Oldenburg, Germany
}

Edited by:

Agnes Gruart, University Pablo de Olavide, Spain

Reviewed by:

Christopher I. Petkov, Newcastle

University, UK

Risto Näätänen, University of

Helsinki, Finland

*Correspondence:

Manuel S. Malmierca, Auditory

Neurophysiology Unit, Laboratory

for the Neurobiology of Hearing,

Institute of Neuroscience of Castilla

y León, University of Salamanca, C/.

Pintor Fernando Gallego,

No. 1, 37007

Salamanca, Spain

e-mail:msm@usal.es
The ability to detect unexpected stimuli in the acoustic environment and determine their behavioral relevance to plan an appropriate reaction is critical for survival. This perspective article brings together several viewpoints and discusses current advances in understanding the mechanisms the auditory system implements to extract relevant information from incoming inputs and to identify unexpected events. This extraordinary sensitivity relies on the capacity to codify acoustic regularities, and is based on encoding properties that are present as early as the auditory midbrain. We review state-of-the-art studies on the processing of stimulus changes using non-invasive methods to record the summed electrical potentials in humans, and those that examine single-neuron responses in animal models. Human data will be based on mismatch negativity (MMN) and enhanced middle latency responses (MLR). Animal data will be based on the activity of single neurons at the cortical and subcortical levels, relating selective responses to novel stimuli to the MMN and to stimulus-specific neural adaptation (SSA). Theoretical models of the neural mechanisms that could create SSA and novelty responses will also be discussed.

Keywords: auditory, potassium channels, regularity, deviance detection, sensory adaptation, stimulus-specific adaptation (SSA), mismatch negativity (MMN), middle latency response (MLR)

\section{INTRODUCTION}

Traditionally, studies on the auditory nervous system have relied on the analysis of neuronal responses to simple stimuli. While this approach has been useful to understand the basic mechanisms that operate in the auditory system, recent studies are using more ecologically valid stimuli to explore the interplay of the different levels of the auditory hierarchy, from the brainstem to the cortex, in a realistic environment. To navigate in an ever-changing reallife scene, the brain is continuously using the sensory past to form expectations about the future.

Most of us have experienced an unbearably loud clock or a busy street that—mysteriously—after a while we stop noticing. Adaptation is a common feature in sensory processing, and the auditory system is not an exception. There are different theories that are not mutually exclusive, about why sensory adaptation is so pervasive. It decreases the amount of information flowing in the system when the stimulus does not vary or is repeated, it increases the sensitivity to detect stimulus changes and abrupt onsets (Ulanovsky et al., 2003; Puccini et al., 2006) and/or it provides a gain control mechanism in the encoding system (Rabinowitz et al., 2011). All these strategies facilitate the detection of novel stimuli, making adaptation and novelty detection closely intertwined.
In order for the nervous system to determine whether a sound is "novel"-in its current context-, there must be some ongoing storage of information about which sounds have already occurred and how they are related to each other. Novelty or unexpectedness can arise from rareness or from abrupt change (as in the above examples), but can also take more complex forms: In some cases (such as when a sound source is moving away from the listener), stimulus change is expected while stimulus repetition is unexpected. The auditory system easily forms expectations at different levels of complexity (Näätänen et al., 2001, 2010); hence the related phenomena are regarded as signs of regularity encoding rather than mere adaptation to an unchanging environment (Winkler, 2007). In this perspective article, we will discuss the neuronal basis and functional implications of this amazing feat of the auditory system.

\section{STIMULUS-SPECIFIC ADAPTATION APPEARS SUBCORTICALLY}

Human electroencephalographic (EEG) studies of responses to sensory stimuli have shown that the waveform elicited by a "novel" (low-probability) stimulus differs from that elicited by a predictable (high-probability) stimulus. Indeed, the detection of "novel" (unexpected) sounds has been associated to a 
particular brain response derived from the human EEG, the mismatch negativity (MMN; Näätänen et al., 1978; for recent review, see Näätänen et al., 2007). The MMN is an auditory event-related brain potential (ERP) component thought to index the mechanisms underlying auditory regularity encoding. MMN can be obtained using an "oddball paradigm", in which a highprobability ("standard") sound is occasionally replaced by a lowprobability ("deviant") sound. Differences in sound probability are created by setting up a sequential regularity; that is, a constraint in the allowed transitions between successive stimuli in the sequence. In the simplest case, this regularity is based on stimulus/feature repetition (cf. Grill-Spector et al., 2006, for a comprehensive account in the visual domain); more complex cases include feature alternation, gradual progression, or feature conjunctions (cf. Näätänen et al., 2001, 2010; Winkler, 2007). MMN is measured as the difference between the ERP elicited by the deviant sound and that elicited by the standard sound and peaks at 150-200 ms from deviation onset (Note that if the standard is defined by repetition, a compensation for the larger afferent responses of the infrequent deviant sound must be introduced; cf. Schröger and Wolff, 1996). MMN can also be recorded in non-human species (e.g., Javitt et al., 1994; Tikhonravov et al., 2008) including rodents (Astikainen et al., 2006; Nakamura et al., 2011; Budd et al., 2012; Shiramatsu et al., 2013).

Because the detection of "novelty" (i.e., simple acoustic deviance or complex regularity violation) requires information storage and comparison over time, it must involve more or less complex memory operations (Näätänen et al., 2001, 2010; Winkler, 2007). For this reason it is commonly believed that novelty detection must be accomplished at the level of the cortex. However, the fact that the MMN persists during sleep or anesthesia suggests that it is "preattentive" in origin (Tiitinen et al., 1994) and therefore could originate subcortically. Although this idea received support from early seminal studies suggesting subcortical generators to the MMN (Kraus et al., 1994; Csépe, 1995), it has been largely unexplored until recently (Escera and Malmierca, 2014). Yet, recent human studies have suggested that subcortical auditory stations may undergoo substantial experiencedependent plasticity (Chandrasekaran and Kraus, 2010; Kraus and Skoe, 2010; Chandrasekaran et al., 2013; Skoe et al., 2013, 2014). For example, Kraus et al. have found that the brainstem activity is enhanced when stimulus sequences contain predictable sounds compared to more random (i.e., unpredictable) sequences that is infrequent and unpredictable. In the present perspective, we will present data to suggest the active role of the inferior colliculus (IC), and in general of the subcortical auditory pathway in regularity encoding and deviance detection". It is even suggested that the IC is analogous to V1 in processing complexity (King and Nelken, 2009). Yet the assumption that novelty detection is a cortical function has persisted, not only for theoretical reasons (complexity of the involved regularities as explained above), but also for technical reasons, because it is difficult to pinpoint the site at which scalp EEG waveforms are generated, especially in the case of subcortical structures.

Importantly, over the past 10 years, a similar phenomenon to that described for MMN has been demonstrated to occur at the cellular level using neurophysiological tools. Single neuron recordings using an oddball paradigm similar to that used for MMN studies have shown a decreased response to a repeated (standard) sound and an increased response to a less repeated (deviant) sound within a sound sequence in the cat auditory cortex (AC; Ulanovsky et al., 2003). This phenomenon has been termed "stimulus-specific adaptation" (SSA) and was originally proposed as the neuronal correlate of MMN. Moreover, it was assumed that SSA was a unique and emerging property of the AC neurons (Ulanovsky et al., 2003; Nelken and Ulanovsky, 2007).

However, there is now a substantial body of evidence challenging this idea (Pérez-González et al., 2005, 2012; Anderson et al., 2009; Malmierca et al., 2009; Yu et al., 2009; Antunes et al., 2010; Antunes and Malmierca, 2011, 2013; Bäuerle et al., 2011; Zhao et al., 2011; Duque et al., 2012, 2014; Patel et al., 2012; Ayala et al., 2013; Ayala and Malmierca, 2013; Pérez-González and Malmierca, 2014). These studies have demonstrated that SSA also occurs subcortically (Figure 1A), i.e., in the IC and medial geniculate body (MGB). Moreover, many aspects of SSA seen in the IC (Ayala and Malmierca, 2013; Pérez-González and Malmierca, 2014) and in the MGB (Antunes et al., 2010; Antunes and Malmierca, 2013) are very similar (Figures 1B,C) to that described in the AC (Ulanovsky et al., 2003, 2004; von der Behrens et al., 2009; Taaseh et al., 2011). However, a major difference between the cortical and subcortical SSA is that SSA in the IC and MGB is stronger in the non-lemniscal divisions, while the first lemniscal nucleus where SSA is strong and widespread is the primary AC (Ulanovsky et al., 2003; Nelken and Ulanovsky, 2007). A simple interpretation would be that SSA is merely imposed upon IC and MGB neurons (Nelken and Ulanovsky, 2007) through the massive descending corticofugal pathways in a top-down fashion (Malmierca and Ryugo, 2011). However, recent work has demonstrated that SSA in the MGB and IC is not merely inherited from the AC (Antunes and Malmierca, 2011; Anderson and Malmierca, 2013) since cortical deactivation does not change SSA sensitivity. Hence SSA may be created independently at each level of the auditory hierarchy (Escera and Malmierca, 2014). For example, the pharmacological manipulation of $\mathrm{GABA}_{\mathrm{A}}$ receptors in the IC (Pérez-González et al., 2012; Pérez-González and Malmierca, 2012) and MGB (Duque et al., 2014) has shown that, while not involved in the generation of SSA, inhibitory inputs could modulate the level of adaptation by reducing the relative strength of the response to the standard and deviant stimuli increasing the deviant to standard ratio, acting as a gain control mechanism, similar to the iceberg effect (Figures 1D,F; Pérez-González et al., 2012). Note that this does not exclude the possibility that the consequences of SSA are also transmitted to the next relay station as in a cascade. In fact, adaptation effects cascade through the visual system (Dhruv and Carandini, 2014).

\section{CELLULAR MECHANISMS OF AUDITORY CORTEX ADAPTATION}

The previous section outlined findings showing that auditory adaptation (often referred to as forward masking/suppression; e.g., Wehr and Zador, 2005; Scholes et al., 2011) can be attributed not only to AC but also to subcortical structures (cf. 
A

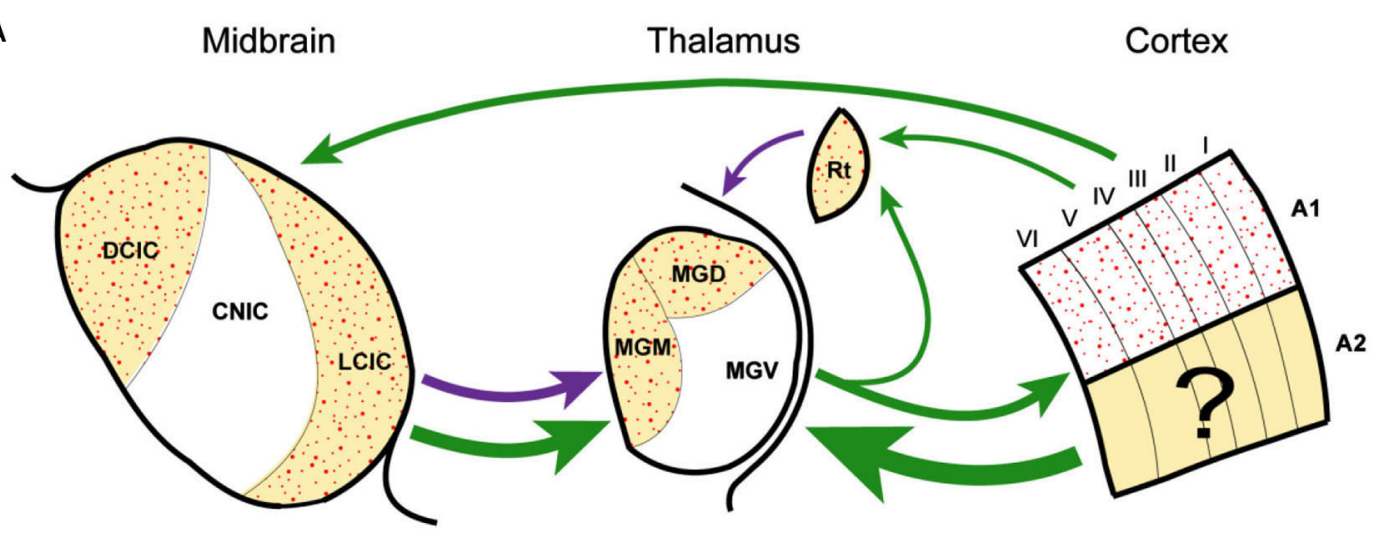

B
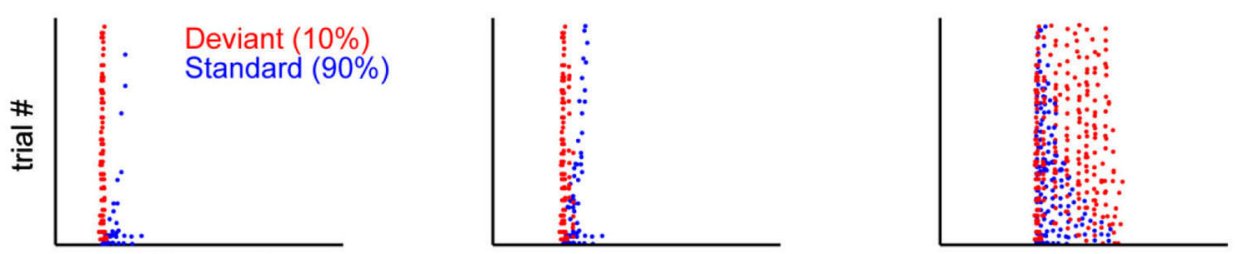

Time (ms)

C
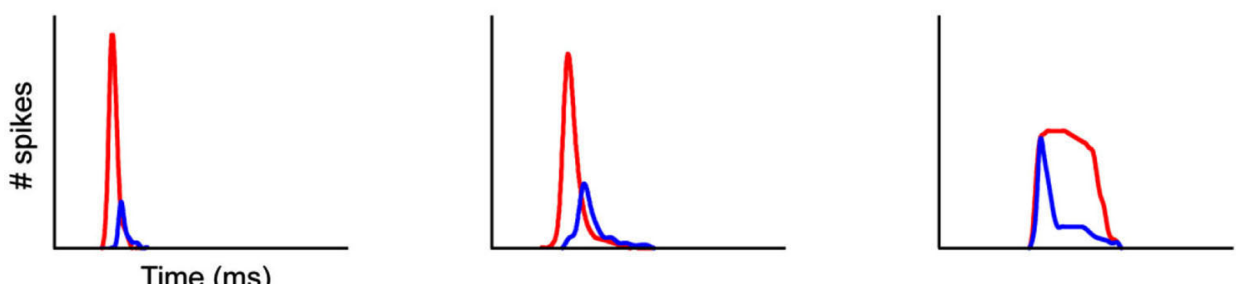

Time (ms)

D
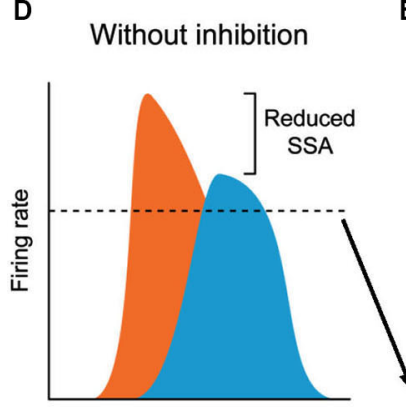

Time (ms)
E

With inhibition

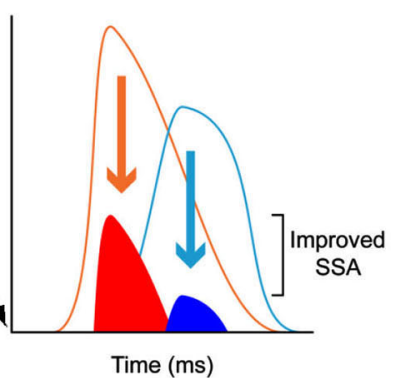

F $\quad$ In vitro silent

- In vitro active

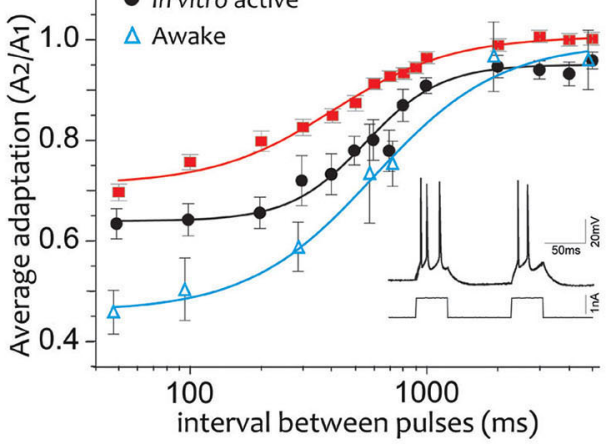

FIGURE 1 | (A) Schematic diagram (after Escera and Malmierca, 2014) illustrating the main anatomical subdivisions as well as the similarities and differences of SSA at the IC (left column), MGB (middle column) and auditory cortex (AC) (right column). Arrows indicate the major connections between these regions. Green arrows are excitatory projections, purple arrows inhibitory connections. Non-lemniscal divisions are highlighted as yellow-shade areas and stipple areas show regions where SSA is strong. Note that SSA is linked to non-lemniscal regions in IC and MGB but to the lemniscal primary AC. Dot raster plots (B) and peri-stimulus histogram (PSTHs); (C) that show the adaptation of the response to the standard stimulus (blue dots) while the response to the deviant stimulus (red dots) does not adapt. (D) Neurons respond to deviants (orange) and standards (light blue) with high firing rates, in the absence of inhibition and thus the deviant to standard ratio is small. By contrast, $\mathrm{GABA}_{A^{-}}$mediated inhibition (E) reduces the responses to both deviants (red) and standards (dark blue) acting as in the "iceberg effect" increasing the deviant to standard ratio and thus enhancing SSA. For more details see Pérez-González et al. (2012). (F) Average adaptation time course in single neurons in the $\mathrm{AC}$ in the awake animal, in silent cortical slices and "active" cortical slices (where the intracellularly recorded neuron was induced to fire following a prerecorded neuron in the awake animal). Note that all adaptation in vitro is due strictly to cellular (and not synaptic) mechanisms. To estimate the time course, two identical $50 \mathrm{~ms}$ pulses of current injection were delivered with intervals spanning from $50 \mathrm{~ms}$ to $5 \mathrm{~s}$ (see inset). The relative frequency rate of the second response with respect to the first one are represented. Note that while the time course is similar, the larger

(Continued) 


\section{FIGURE 1 | Continued}

adaptation is that in the awake animal and the least the one in the silent slice. Part of the difference between those two is due to the ongoing activity in the awake, as the in vitro "active" preparation indicates. A logistic function was fitted. Error bars are SEM. For more details see Abolafia et al. (2011). Abbreviations: A1, primary auditory cortex; A2 non-primary auditory cortex; CNIC, central nucleus of the inferior colliculus; DCIC, dorsal cortex of the inferior colliculus; LCIC, lateral cortex of the inferior colliculus; MGD dorsal division of the medial geniculate body; MGM, medial division of the medial geniculate body; MGV, ventral division of the medial geniculate body; Rt, reticular thalamic nucleus.

Pérez-González and Malmierca, 2014). Besides this anatomical diversity, adaptation shows diversity as well in the temporal dimension: it occurs at different time scales spanning many orders of magnitude, from several milliseconds to tens of seconds (Ulanovsky et al., 2004; Costa-Faidella et al., 2011). This broad time range makes it compatible with the participation of different potential underlying mechanisms. Some of these are synaptic mechanisms such as: synaptic depression (Wehr and Zador, 2005), decreased excitation or lateral inhibition (Qin and Sato, 2004), increased inhibition (Zhang et al., 2003), or excitatory/inhibitory imbalance (De Ribaupierre et al., 1972; Volkov and Galazjuk, 1991; Ojima and Murakami, 2002; Oswald et al., 2006).

Paradoxically, the role of intrinsic membrane mechanisms such as potassium currents in AC adaptation had been ruled out or neglected until recently, even though they participate in sensory adaptation in other sensory cortices: sensorimotor (Schwindt et al., 1988), barrel (Diaz-Quesada and Maravall, 2008), or visual cortex (Sanchez-Vives et al., 2000a,b; Wang et al., 2003). Potassium currents act as an activity-dependent adaptation mechanism, such that depolarization and high frequency firing during sensory responses induce not only the activation of voltage-dependent potassium currents but also an intracellular increase of ions like $\mathrm{Ca}^{2+}$ and $\mathrm{Na}^{+}$that activate iondependent $\mathrm{K}^{+}$channels (for reviews see Sah and Faber, 2002; Bhattacharjee and Kaczmarek, 2005). Even if a synaptic depolarization is subthreshold, sodium entering through glutamate receptors can activate sodium-dependent $\mathrm{K}^{+}$channels (Nanou et al., 2008). The activation of potassium currents hyperpolarizes the membrane potential, decreasing the neuronal responsiveness to subsequent inputs with time courses that range from tens of milliseconds to tens of seconds. Such slow time courses are supported by the dynamics of the relevant intracellular ions -in particular $\mathrm{Na}^{+}-$in the vicinity of $\mathrm{K}^{+}$channels and their binding/unbiding to them. Sodium and calcium-dependent potassium currents also exist in neurons of the rat AC (Abolafia et al., 2011).

In order to explore whether potassium channels do actually play a role in auditory adaptation in the awake animal, it was first necessary to determine the time course of adaptation of AC neurons in chronically implanted awake rats. In a recent study, Abolafia et al. (2011) delivered two auditory stimuli separated by intervals ranging between milliseconds and several seconds. The attenuation of the response to the second stimulus with respect to the first along time provided the time course of adaptation, showing that a $50 \mathrm{~ms}$ sound in the awake animal influences responses occurring up to 2-5 s later. The same auditory protocol was then mimicked in the cortical neurons in vitro (Figure 1F) through intracellular current injections that had the same duration and would evoke the same number of spikes as the auditory stimuli did in the awake animal. It should be noted that those were silent slices and therefore all the observed phenomena only involve the intracellularly recorded neuron and not the network in which it is embedded. This approach demonstrated that the time course of adaptation observed in the awake rat was highly similar to the one detected in vitro (Abolafia et al., 2011). However, the response attenuation was larger in the awake animal. A critical factor contributing to this difference is the spontaneous ongoing activity in the awake animal, that builds up adaptation currents contributing to a steady state of adaptation in an active (awake) cortical network with respect to a silent one (slice). This steady state of adaptation has been demonstrated by replicating spontaneous firing recorded from neurons in the awake animal in in vitro neurons by means of intracellular current injection (Figure 7; Abolafia et al., 2011).

Therefore, a significant fraction of cortical auditory adaptation can be explained just by intrinsic cellular mechanisms ( $\mathrm{K}^{+}$channels), although this does not exclude the additional participation of synaptic mechanisms. Hence ionic mechanisms should be incorporated into mechanistic explanations of adaptation and novelty detection. Further, the interaction between potassium channels-mediated adaptation and synaptic depression provides computational capabilities to the network to detect rate of change, anticipation and detection of novelty (Puccini et al., 2006, 2007).

An argument that is often used against ionic channels as a mechanism for auditory adaptation is that they lack input specificity and thus they would attenuate any incoming input, not supporting the delicate SSA (Ulanovsky et al., 2003, 2004; Wehr and Zador, 2005). However, based on cortical circuitry, a speculation would be that cellular hyperpolarization and decreased excitability are cellular properties that become network properties by reverberating in the local recurrent circuitry of the cortical column, contributing to adaptation in neurons with the same frequency-specificity.

\section{NOVELTY DETECTION IN A CORTICO-SUBCORTICAL DISTRIBUTED CEREBRAL NETWORK}

The idea that novelty detection and the underlying regularity encoding can take place at levels hierarchically lower/earlier than those generating the MMN, is also supported by a series of human studies that recorded middle latency responses (MLRs) in the oddball paradigm. The MLR is a series of characteristic waveforms elicited to discrete auditory stimuli in the range $12-50 \mathrm{~ms}$ postonset (Figure 2A). They are labeled as N0, P0, Na, Pa, and $\mathrm{Nb}$ (sometimes $\mathrm{Pb}$, equivalent to $\mathrm{P} 50$, is included), and represent the earliest cortical responses to a sound (Winkler et al., 2013). For example, it has been shown that the P0 waveform peaking at 16$19 \mathrm{~ms}$ is generated in primary AC, whereas subsequent components are generated in surrounding areas of the supratemporal plane and gyrus (Yvert et al., 2001, 2005). 

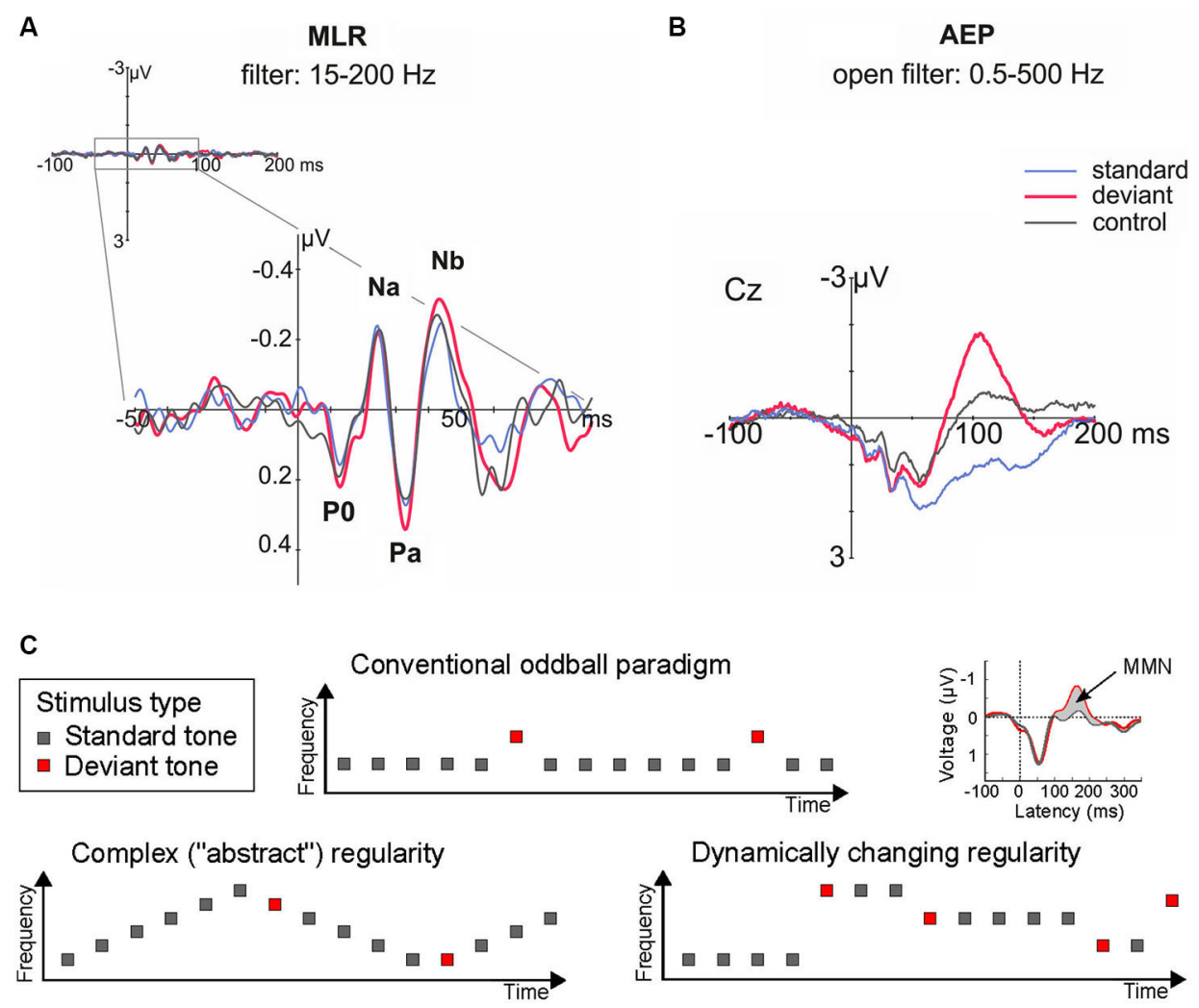

FIGURE 2 | (A-B) Human auditory evoked potentials in the latency range of the middle latency response (MLR, at circa 20-50 ms; A, left), and later on, by the long latency response (circa 100-200 ms; B, right), reveal that deviance detection based on regularity encoding takes place in human $\mathrm{AC}$ at recurrent neural networks; red, deviant response; blue, standard response; black, contro response. Note that physically identical sounds elicited larger responses when they were presented as deviant than as standard or control stimuli in the MLR (by the $\mathrm{Nb}$ component, at $40 \mathrm{~ms}$ from change onset) and long-latency (i.e., MMN, by 100 ms) ranges. Adapted from Grimm et al. (2011). (C) Schematic illustration of stimulus paradigms departing from the standard oddball paradigm to achieve an increase in complexity and ecological validity. These and similar paradigm variations have shown that complex regularities in the acoustic environment can be extracted from just a few exemplars, as demonstrated by long-latency auditory evoked potentials (the MMN).
One seminal study investigating novelty detection in the MLR time range (Grimm et al., 2011) used a frequency "deviant" tone of $1200 \mathrm{~Hz}$ presented amongst $800 \mathrm{~Hz}$ standard tones, and implemented a "reversed" condition (where deviant and standard switched their roles, controlling for stimulus-feature effects), and a "controlled" condition (where the deviant was embedded amongst a series of other equiprobable tones, controlling for refractoriness effects; cf. Schröger and Wolff, 1996). The results (Figure 2A) showed an enhanced Nb MLR component elicited by the deviant tones when compared both to the reversed standard and to the control stimulus. This reveals that "true" deviance detection (i.e., based on regularity encoding rather than simple adaptation) occurs at latencies as short as $40 \mathrm{~ms}$ from sound onset (Grimm et al., 2011). This demonstration of regularity-based deviance detection is important to develop a general framework encompassing not only simple adaptation phenomena (i.e., based on stimulus repetition; cf. Grill-Spector et al., 2006), but also more complex forms of auditory regularity encoding.

Subsequent studies confirmed the involvement of the $\mathrm{Nb}$ in frequency deviant detection (Alho et al., 2012; Leung et al., 2012; Althen et al., 2013), and revealed that other MLR waveforms are related to deviant detection in other auditory stimulus features, such as the Na for location changes (Sonnadara et al., 2006; Cornella et al., 2012; Grimm et al., 2012), and the transition between the Na-Pa waveforms for intensity changes (Althen et al., 2011) or the Pa-Nb for temporal deviations (Leung et al., 2013).

A further magnetoencephalographic (MEG) study revealed that deviant-detection is in fact a distributed property of the AC, with neural populations generating its $\mathrm{Nb} / \mathrm{MLR}$ correlates located in more medial and anterior regions than those giving rise to the MMN (Recasens et al., 2014a). Hence, these early deviancerelated responses by the MLR (Figure 2A) may represent, by their earlier latency and lower hierarchical distribution, a better human psychophysiological correlate of the single-unit novelty responses than the MMN (Figure 2B; Escera et al., 2013).

At lower auditory stations, while no correlates of deviance detection were found in the auditory brainstem response (ABR; Slabu et al., 2010; Althen et al., 2011), a recent study that measured the frequency following response (FFR; Skoe and Kraus, 2010) revealed the involvement of the human IC in deviance detection based on regularity encoding (Slabu et al., 2012) as in animal studies (Gao et al., 2014). 
Interestingly, studies addressing whether more complex types of auditory regularities could be encoded at the level of the MLR-generating system, such as tone alternation (Cornella et al., 2012), feature conjunction (Althen et al., 2013) or pattern regularities (Recasens et al., unpublished observations), yielded negative results. These negative findings, together with the early effects described above for simple feature violations, suggest that the auditory system is organized in a hierarchical fashion, so that complex regularities require higher levels of the auditory hierarchy to be picked up (Grimm and Escera, 2012; Escera and Malmierca, 2014). However, it should be noted that preliminary studies using single unit recordings in animals suggest that IC neurons show sensitivity to complex regularities (Aguillon et al., 2013).

\section{REGULARITY ENCODING AND NOVELTY DETECTION IN COMPLEX STIMULUS ENVIRONMENTS}

Now that the existence of "novelty responses" along the auditory pathway is well established both in animal and human studies, an important next step will be to characterize the properties of this mechanism in more detail-particularly with more ecologically valid stimulus configurations (Figure 2C). Initial demonstrations of these effects have often used repetitive sound sequences over long recording periods, making it difficult to distinguish between simple (repetition-based) adaptation and a "true" regularity encoding account. But realistic sound sources exhibit more complex forms of organization; for instance, they do not repeat their sound emissions but change them in a regular manner (e.g., when gradually moving to a different location). There is now abundant evidence to show that the MMN responds to violations of such complex forms of regularities (for reviews, see Näätänen et al., 2001, 2010; Winkler, 2007). Whether the same is true at earlier levels of the auditory hierarchy is still a matter of investigation (see above; cf. Cornella et al., 2012; Aguillon et al., 2013; Althen et al., 2013). It is important to resolve this issue to develop better links between the neuronal models reviewed above and theoretical accounts of regularity encoding coming from MMN-based investigations (Winkler, 2007) or from closely related research fields such as predictive coding (Friston, 2005).

Another form of real-life complexity has received much less attention: a sound source usually does not exhibit the same type of regular behavior over hours, but behaves according to one regularity for a while and then according to another regularity (e.g., it stands still, gradually changes location, then stands still again). Hence the brain should be able to extract regularities considerably faster than shown in a typical protocol where the same regularity is valid for an hour or longer. Some MMN studies have demonstrated that regularity extraction is possible from just a few exemplars (Cowan et al., 1993; Winkler et al., 1996; Huotilainen et al., 2001; Haenschel et al., 2005; Bendixen et al., 2007, 2008). Both human and animal data suggest that the brain picks up regularities at multiple timescales (Ulanovsky et al., 2004; CostaFaidella et al., 2011). This is important because it demonstrates quick adaptation to newly emerging regularities as well as longerlasting impact of previously valid regularities, both of which appear to be ecologically adaptive. Systematic investigations along these lines across different levels of the auditory hierarchy should prove informative for a comprehensive model of regularity encoding and novelty detection.

Characterizing the mechanisms and temporal dynamics of regularity encoding is important also in the face of its implications for other cognitive processes. Sensory regularities render the environment predictable and hence serve preparation of appropriate motor responses. Another benefit of regularity encoding may lie within perception itself: Regularities have been shown to support auditory scene analysis, i.e., disentangling a mixture of overlapping signals emitted by several concurrently active sound sources (see Bendixen, 2014, for review). Furthermore, the ability to use certain regularities for sound source segregation appears to decline with age (Rimmele et al., 2012), possibly linked with an age-related decline in regularity encoding capacities (Pekkonen, 2000; Näätänen et al., 2012). Hence a comprehensive understanding of regularity encoding and novelty detection, as well as of impairments in these processes, may shed light on crucial aspects of everyday listening experience.

\section{CONCLUDING REMARKS}

In the auditory system, the sequence of previous sensory stimulation and its resulting neuronal activation deeply influence subsequent responses-providing the ground for auditory adaptation, regularity encoding, and novelty detection. The anatomical stages and mechanisms contributing to these phenomena can now be partly delineated based on recent advances outlined above. Animal studies help to shed light on the neurophysiological mechanisms subserving adaptation. We have argued that not only synaptic but also cellular mechanisms should be taken into account as viable contributors. Furthermore, we suggested that it may be more appropriate to link SSA with MLR deviance-related effects than with the MMN component.

Human studies are contributing to a more comprehensive picture of the mechanisms involved in auditory perception where regularity encoding seems to be paramount. Moreover, human ERP studies provide a link to clinical neuroscience so that pathophysiological mechanisms of auditory information processing in neurological, psychiatric and developmental disorders can be revealed (Näätänen et al., 2011, 2012). In this regard, the recently discovered correlates of auditory deviance detection by the MLR latency range, and even the psychophysiological subcortical correlates revealed by the FFR may help to go a step beyond the $\mathrm{MMN}$ in understanding these disorders. Animal correlates again provide a valuable complementary approach to elucidate the role of novelty and adaptation in auditory perception under normal and pathological conditions. Additional insight may be gained by studying animal models in the same way as done in human studies (e.g., MLR, MMN). With this perspective article, we hope to contribute to further promising research at this fascinating intersection.

\section{ACKNOWLEDGMENTS}

We thank Dr. Mel Slater for critical reading of a previous version of the manuscript. This work was supported by the Spanish Ministry of Economy and Knowledge (project BFU201343608-P to Manuel S. Malmierca, project PSI2012-37174 to 
Carles Escera, project BFU2011-27094 to Maria V. SanchezVives, project EUI2009-04083 to Manuel S. Malmierca and project EUI2009-04086 to Carles Escera in the frame of the ERANET NEURON) and the Catalan Government (SGR2014177 to Carles Escera, SGR2009-1363 to Maria V. Sanchez-Vives). Carles Escera is also supported by the ICREA Academia Distinguished Professorship. Alexandra Bendixen is supported by the German Research Foundation (DFG Cluster of Excellence 1077 "Hearing4all").

\section{REFERENCES}

Abolafia, J. M., Vergara, R., Arnold, M. M., Reig, R., and Sanchez-Vives, M. V. (2011). Cortical auditory adaptation in the awake rat and the role of potassium currents. Cereb. Cortex 21, 977-990. doi: 10.1093/cercor/bhq163

Aguillon, B. E. N., Nieto, J., Escera, C., and Malmierca, M. S. (2013). Response to complex patterns of regularity in the inferior colliculus of the anesthetized rat. Abst ARO meeting \#663, 36, 321-322.

Alho, K., Grimm, S., Mateo-León, S., Costa-Faidella, J., and Escera, C. (2012). Early processing of pitch in the human auditory system. Eur. J. Neurosci. 36, 29722978. doi: 10.1111/j.1460-9568.2012.08219.x

Althen, H., Grimm, S., and Escera, C. (2011). Fast detection of unexpected sound intensity decrements as revealed by human evoked potentials. PLoS One 6:e28522. doi: 10.1371/journal.pone.0028522

Althen, H., Grimm, S., and Escera, C. (2013). Simple and complex acoustic regularities are encoded at different levels of the auditory hierarchy. Eur. J. Neurosci. 38, 3448-3455. doi: 10.1111/ejn.12346

Anderson, L. A., and Malmierca, M. S. (2013). The effect of auditory cortex deactivation on stimulus-specific adaptation in the inferior colliculus of the rat. Eur. J. Neurosci. 37, 52-62. doi: 10.1111/ejn.12018

Anderson, L. A., Christianson, G. B., and Linden, J. F. (2009). Stimulus-specific adaptation occurs in the auditory thalamus. J. Neurosci. 29, 7359-7363. doi: 10. 1523/JNEUROSCI.0793-09.2009

Antunes, F. M., and Malmierca, M. S. (2011). Effect of auditory cortex deactivation on stimulus-specific adaptation in the medial geniculate body. J. Neurosci. 31, 17306-17316. doi: 10.1523/JNEUROSCI.1915-11.2011

Antunes, F. M., and Malmierca, M. S. (2013). An overview of stimulus-specific adaptation in the auditory thalamus. Brain Topogr. doi: 10.1007/s10548-0130342-6. [Epub ahead of print].

Antunes, F. M., Nelken, I., Covey, E., and Malmierca, M. S. (2010). Stimulusspecific adaptation in the auditory thalamus of the anesthetized rat. PLoS One 5:e14071. doi: 10.1371/journal.pone.0014071

Astikainen, P., Ruusuvirta, T., Wikgren, J., and Penttonen, M. (2006). Memorybased detection of rare sound feature combinations in anesthetized rats. Neuroreport 17, 1561-1564. doi: 10.1097/01.wnr.0000233097.13032.7d

Ayala, Y. A., and Malmierca, M. S. (2013). Stimulus-specific adaptation and deviance detection in the inferior colliculus. Front. Neural Circuits 6:89. doi: 10 . 3389/fncir.2012.00089

Ayala, Y. A., Perez-Gonzalez, D., Duque, D., Nelken, I., and Malmierca, M. S. (2013). Frequency discrimination and stimulus deviance in the inferior colliculus and cochlear nucleus. Front. Neural Circuits 6:119. doi: 10.3389/fncir.2012.00119

Bäuerle, P., von der Behrens, W., Kössl, M., and Gaese, B. H. (2011). Stimulusspecific adaptation in the gerbil primary auditory thalamus is the result of a fast frequency-specific habituation and is regulated by the corticofugal system. J. Neurosci. 31, 9708-9722. doi: 10.1523/JNEUROSCI.5814-10.2011

Bendixen, A. (2014). Predictability effects in auditory scene analysis: a review. Front. Neurosci. 8:60. doi: 10.3389/fnins.2014.00060

Bendixen, A., Prinz, W., Horváth, J., Trujillo-Barreto, N. J., and Schröger, E. (2008). Rapid extraction of auditory feature contingencies. Neuroimage 41, 1111-1119. doi: 10.1016/j.neuroimage.2008.03.040

Bendixen, A., Roeber, U., and Schröger, E. (2007). Regularity extraction and application in dynamic auditory stimulus sequences. J. Cogn. Neurosci. 19, 1664 1677. doi: 10.1162/jocn.2007.19.10.1664

Bhattacharjee, A., and Kaczmarek, L. K. (2005). For $\mathrm{K}^{+}$channels, $\mathrm{Na}^{+}$is the new $\mathrm{Ca}^{2+}$. Trends Neurosci. 28, 422-428. doi: 10.1016/j.tins.2005.06.003

Budd, T. W., Nakamura, T., Fulham, W. R., Todd, J., Schall, U., Hunter, M., et al. (2012). Repetition suppression of the rat auditory evoked potential at brief stimulus intervals. Brain Res. 1498, 59-68. doi: 10.1016/j.brainres. 2012.12.033

Chandrasekaran, B., and Kraus, N. (2010). The scalp-recorded brainstem response to speech: neural origins and plasticity. Psychophysiology 47, 236-246. doi: 10. 1111/j.1469-8986.2009.00928.x

Chandrasekaran, B., Skoe, E., and Kraus, N. (2013). An integrative model of subcortical auditory plasticity. Brain Topogr. doi: 10.1007/s10548-013-0323-9. [Epub ahead of print].

Cornella, M., Leung, S., Grimm, S., and Escera, C. (2012). Detection of simple and pattern regularity violations occurs at different levels of the auditory hierarchy. PLoS One 7:e43604. doi: 10.1371/journal.pone.0043604

Costa-Faidella, J., Grimm, S., Slabu, L., Díaz-Santaella, F., and Escera, C. (2011). Multiple time scales of adaptation in the auditory system as revealed by human evoked potentials. Psychophysiology 48, 774-783. doi: 10.1111/j.1469-8986.2010. 01144.x

Cowan, N., Winkler, I., Teder, W., and Näätänen, R. (1993). Memory prerequisites of mismatch negativity in the auditory event-related potential (ERP). J. Exp. Psychol. Learn. Mem. Cogn. 19, 909-921. doi: 10.1037/0278-7393.19.4.909

Csépe, V. (1995). On the origin and development of the mismatch negativity. Ear Hear. 16, 91-104. doi: 10.1097/00003446-199502000-00007

De Ribaupierre, F., Goldstein, M. H. Jr., and Yeni-Komshian, G. (1972). Intracellular study of the cat's primary auditory cortex. Brain Res. 48, 185-204. doi: 10. 1016/0006-8993(72)90178-3

Dhruv, N. T., and Carandini, M. (2014). Cascaded effects of spatial adaptation in the early visual system. Neuron 81, 529-535. doi: 10.1016/j.neuron.2013.11.025

Diaz-Quesada, M., and Maravall, M. (2008). Intrinsic mechanisms for adaptive gain rescaling in barrel cortex. J. Neurosci. 28, 696-710. doi: 10. 1523/JNEUROSCI.4931-07.2008

Duque, D., Malmierca, M. S., and Caspary, D. M. (2014). Modulation of stimulusspecific adaptation by GABAA receptor activation or blockade in the medial geniculate body of the anesthetized rat. J. Physiol. 592, 729-743. doi: 10. 1113/jphysiol.2013.261941

Duque, D., Perez-Gonzalez, D., Ayala, Y. A., Palmer, A. R., and Malmierca, M. S. (2012). Topographic distribution, frequency and intensity dependence of stimulus-specific adaptation in the inferior colliculus of the rat. J. Neurosci. 32, 17762-17774. doi: 10.1523/JNEUROSCI.3190-12.2012

Escera, C., and Malmierca, M. S. (2014). The auditory novelty system: an attempt to integrate human and animal research. Psychophysiology 51, 111-123. doi: 10. 1111/psyp.12156

Escera, C., Leung, S., and Grimm, S. (2013). Deviance detection based on regularity encoding along the auditory hierarchy: electrophysiological evidence in humans. Brain Topogr. doi: 10.1007/s10548-013-0328-4. [Epub ahead of print].

Friston, K. (2005). A theory of cortical responses. Philos. Trans. R. Soc. Lond. B Biol. Sci. 360, 815-836. doi: 10.1098/rstb.2005.1622

Gao, P. P., Zhang, J. W., Cheng, J. S., Zhou, I. Y., and Wu, E. X. (2014). The inferior colliculus is involved in deviant sound detection as revealed by BOLD fMRI. Neuroimage 91, 220-227. doi: 10.1016/j.neuroimage.2014.01.043

Grill-Spector, K., Henson, R., and Martin, A. (2006). Repetition and the brain: neural models of stimulus-specific effects. Trends Cogn. Sci. 95, 995-1007. doi: 10.1016/j.tics.2005.11.006

Grimm, S., and Escera, C. (2012). Auditory deviance detection revisited: evidence for a hierarchical novelty system. Int. J. Psychophysiol. 85, 88-92. doi: 10.1016/j. ijpsycho.2011.05.012

Grimm, S., Escera, C., Slabu, L. M., and Costa-Faidella, J. (2011). Electrophysiological evidence for the hierarchical organization of auditory change detection in the human brain. Psychophysiology 48, 377-384. doi: 10.1111/j.1469-8986.2010. 01073.x

Grimm, S., Recasens, M., Althen, H., and Escera, C. (2012). Ultrafast tracking of sound location changes as revealed by human auditory evoked potentials. Biol. Psychol. 89, 232-239. doi: 10.1016/j.biopsycho.2011.10.014

Haenschel, C., Vernon, D. J., Dwivedi, P., Gruzelier, J. H., and Baldeweg, T. (2005). Event- related brain potential correlates of human auditory sensory memory-trace formation. J. Neurosci. 25, 10494-10501. doi: 10.1523/jneurosci. 1227-05.2005

Huotilainen, M., Kujala, A., and Alku, P. (2001). Long-term memory traces facilitate short-term memory trace formation in audition in humans. Neurosci. Lett. 310, 133-136. doi: 10.1016/s0304-3940(01)02096-1

Javitt, D. C., Steinschneider, M., Schroeder, C. E., Vaughan, H. G. Jr., and Arezzo, J. C. (1994). Detection of stimulus deviance within primate primary auditory 
cortex: intracortical mechanisms of mismatch negativity (MMN) generation. Brain Res. 667, 192-200. doi: 10.1016/0006-8993(94)91496-6

King, A., and Nelken, I. (2009). Unraveling the principles of auditory cortical processing: can we learn from the visual system? Nat. Neurosci. 12, 698-701. doi: $10.1038 / \mathrm{nn} .2308$

Kraus, N., and Skoe, E. (2010). Auditory brain stem response to complex sounds: a tutorial. Ear Hear. 31, 302-324. doi: 10.1097/AUD.0b013e3181cdb272

Kraus, N., McGee, T., Littman, T., Nicol, T., and King, C. (1994). Nonprimary auditory thalamic representation of acoustic change. J. Neurophysiol. 72, 12701277.

Leung, S., Cornella, M., Grimm, S., and Escera, C. (2012). Is fast auditory change detection feature-specific? An electrophysiological study in humans. Psychophysiology 49, 933-942. doi: 10.1111/j.1469-8986.2012.01375.x

Leung, S., Recasens, M., Grimm, S., and Escera, C. (2013). Electrophysiological index of acoustic temporal regularity violation in the middle latency range. Clin. Neurophysiol. 124, 2397-2405. doi: 10.1016/j.clinph.2013.06.001

Malmierca, M. S., and Ryugo, D. K. (2011). "Descending connections of auditory cortex to the midbrain and brainstem," in The Auditory Cortex, eds J. A. Winer and C. E. Schreiner (New York, NY: Springer), 189-208.

Malmierca, M. S., Cristaudo, S., Pérez-González, D., and Covey, E. (2009). Stimulus-specific adaptation in the inferior colliculus of the anesthetized rat. J. Neurosci. 29, 5483-5493. doi: 10.1523/JNEUROSCI.4153-08.2009

Näätänen, R., Astikainen, P., Ruusuvirta, T., and Huotilainen, M. (2010). Automatic auditory intelligence: an expression of the sensory-cognitive core of cognitive processes. Brain Res. Rev. 64, 123-136. doi: 10.1016/j.brainresrev.2010. 03.001

Näätänen, R., Gaillard, A. W. K., and Mäntysalo, S. (1978). Early selective-attention effect on evoked potential reinterpreted. Acta Psychol. (Amst). 42, 313-329. doi: 10.1016/0001-6918(78)90006-9

Näätänen, R., Kujala, T., Escera, C., Baldeweg, T., Kreegipuu, K., Carlson, S., et al. (2012). The Mismatch Negativity (MMN) - a unique window to disturbed central auditory processing in aging and different clinical conditions. Clin. Neurophysiol. 123, 424-458. doi: 10.1016/j.clinph.2011.09.020

Näätänen, R., Kujala, T., Kreegipuu, K., Carlson, S., Escera, C., Baldeweg, T., et al. (2011). The mismatch negativity: an index of cognitive decline in neuropsychiatric and neurological diseases and in aging. Brain 134, 3435-3453. doi: 10. 1093/brain/awr064

Näätänen, R., Paavilainen, P., Rinne, T., and Alho, K. (2007). The mismatch negativity $(\mathrm{MMN})$ in basic research of central auditory processing: a review. Clin. Neurophysiol. 118, 2544-2590. doi: 10.1016/j.clinph.2007.04.026

Näätänen, R., Tervaniemi, M., Sussman, E., Paavilainen, P., and Winkler, I. (2001). 'Primitive intelligence' in the auditory cortex. Trends Neurosci. 24, 283-288. doi: 10.1016/s0166-2236(00)01790-2

Nakamura, T., Michie, P. T., Fulham, W. R., Todd, J., Budd, T. W., Schall, U., et al. (2011). Epidural auditory event-related potentials in the rat to frequency and duration deviants: evidence of mismatch negativity? Front. Psychol. 2:367. doi: 10.3389/fpsyg.2011.00367

Nanou, E., Kyriakatos, A., Bhattacharjee, A., Kaczmarek, L. K., Paratcha, G., and El Manira, A. (2008). Na+-mediated coupling between AMPA receptors and KNa channels shapes synaptic transmission. Proc. Natl. Acad. Sci. U S A 105, 2094120946. doi: 10.1073/pnas.0806403106

Nelken, I., and Ulanovsky, N. (2007). Mismatch negativity and stimulus-specific adaptation in animal models. J. Psychophysiol. 21, 214-223. doi: 10.1027/02698803.21.34.214

Ojima, H., and Murakami, K. (2002). Intracellular characterization of suppressive responses in supragranular pyramidal neurons of cat primary auditory cortex in vivo. Cereb. Cortex 12, 1079-1091. doi: 10.1093/cercor/12.10.1079

Oswald, A. M., Schiff, M. L., and Reyes, A. D. (2006). Synaptic mechanisms underlying auditory processing. Curr. Opin. Neurobiol. 16, 371-376. doi: 10. 1016/j.conb.2006.06.015

Patel, C. R., Redhead, C., Cervi, A. L., and Zhang, H. (2012). Neural sensitivity to novel sounds in the rat's dorsal cortex of the inferior colliculus as revealed by evoked local field potentials. Hear. Res. 286, 41-54. doi: 10.1016/j.heares.2012. 02.007

Pekkonen, E. (2000). Mismatch negativity in aging and in Alzheimer's and Parkinson's diseases. Audiol. Neurootol. 5, 216-224. doi: 10.1159/000013883

Pérez-González, D., and Malmierca, M. S. (2012). Variability of the time course of stimulus-specific adaptation in the inferior colliculus. Front. Neural Circuits 6:107. doi: 10.3389/fncir.2012.00107
Pérez-González, D., and Malmierca, M. S. (2014). Adaptation in the auditory system: an overview. Front. Integr. Neurosci. 8:19. doi: 10.3389/fnint.2014.00019

Pérez-González, D., Hernández, O., Covey, E., and Malmierca, M. S. (2012). $\mathrm{GABA}_{\mathrm{A}}$-mediated inhibition modulates stimulus-specific adaptation in the inferior colliculus. PLoS One 7:e34297. doi: 10.1371/journal.pone.0034297

Pérez-González, D., Malmierca, M. S., and Covey, E. (2005). Novelty detector neurons in the mammalian auditory midbrain. Eur. J. Neurosci. 22, 2879-2885. doi: 10.1111/j.1460-9568.2005.04472.x

Puccini, G. D., Sanchez-Vives, M. V., and Compte, A. (2006). Selective detection of abrupt input changes by integration of spike-frequency adaptation and synaptic depression in a computational network model. J. Physiol. Paris 100, 1-15. doi: 10.1016/j.jphysparis.2006.09.005

Puccini, G. D., Sanchez-Vives, M. V., and Compte, A. (2007). Integrated mechanisms of anticipation and rate-of-change computations in cortical circuits. PLoS Comput. Biol. 3:e82. doi: 10.1371/journal.pcbi.0030082

Qin, L., and Sato, Y. (2004). Suppression of auditory cortical activities in awake cats by pure tone stimuli. Neurosci. Lett. 365, 190-194. doi: 10.1016/j.neulet.2004. 04.092

Rabinowitz, N. C., Willmore, B. D., Schnupp, J. W., and King, A. J. (2011). Contrast gain control in auditory cortex. Neuron 70, 1178-1191. doi: 10.1016/j.neuron. 2011.04.030

Recasens, M., Grimm, S., Capilla, A., Nowak, R., and Escera, C. (2014a). Two sequential processes of change detection in hierarchically ordered areas of the human auditory cortex. Cereb. Cortex 24, 143-153. doi: 10.1093/cercor/bhs295

Rimmele, J. M., Schröger, E., and Bendixen, A. (2012). Age-related changes in the use of regular patterns for auditory scene analysis. Hear. Res. 289, 98-107. doi: 10.1016/j.heares.2012.04.006

Sah, P., and Faber, E. S. L. (2002). Channels underlying neuronal calciumactivated potassium currents. Progr. Neurobiol. 66, 345-353. doi: 10.1016/s03010082(02)00004-7

Sanchez-Vives, M. V., Nowak, L. G., and McCormick, D. A. (2000a). Cellular mechanisms of long-lasting adaptation in visual cortical neurons in vitro. J. Neurosci. 20, 4286-4299.

Sanchez-Vives, M. V., Nowak, L. G., and McCormick, D. A. (2000b). Membrane mechanisms underlying contrast adaptation in cat area 17 in vivo. J. Neurosci. 20, 4267-4285.

Scholes, C., Palmer, A. R., and Sumner, C. J. (2011). Forward suppression in the auditory cortex is frequency-specific. Eur. J. Neurosci. 33, 1240-1251. doi: 10. 1111/j.1460-9568.2010.07568.x

Schröger, E., and Wolff, C. (1996). Mismatch response to changes in sound location. Neuroreport 7, 3005-3008. doi: 10.1097/00001756-19961125000041

Schwindt, P. C., Spain, W. J., Foehring, R. C., Stafstrom, C. E., Chubb, M. C., and Crill, W. E. (1988). Multiple potassium conductances and their functions in neurons from cat sensorimotor cortex in vitro. J. Neurophysiol. 59, 424-449.

Shiramatsu, T. I., Kanzaki, R., and Takahashi, H. (2013). Cortical mapping of mismatch negativity with deviance detection property in rat. PLoS One 8:e82663. doi: 10.1371/journal.pone.0082663

Skoe, E., and Kraus, N. (2010). Auditory brainstem response to complex sounds: a tutorial. Ear Hear. 31, 302-324. doi: 10.1097/aud.0b013e3181cdb272

Skoe, E., Chandrasekaran, B., Spitzer, E. R., Wong, P. C., and Kraus, N. (2014). Human brainstem plasticity: the interaction of stimulus probability and auditory learning. Neurobiol. Learn. Mem. 109, 82-93. doi: 10.1016/j.nlm.2013. 11.011

Skoe, E., Krizman, J., Spitzer, E., and Kraus, N. (2013). The auditory brainstem is a barometer of rapid auditory learning. Neuroscience 243, 104-114. doi: 10.1016/j. neuroscience.2013.03.009

Slabu, L. M., Escera, C., Grimm, S., and Costa-Faidella, J. (2010). Early change detection in humans as revealed by auditory brainstem and middle-latency evoked potentials. Eur. J. Neurosci. 32, 859-865. doi: 10.1111/j.1460-9568.2010. 07324.x

Slabu, L., Grimm, S., and Escera, C. (2012). Novelty detection in the human auditory brainstem. J. Neurosci. 32, 1447-1452. doi: 10.1523/jneurosci.2557-11. 2012

Sonnadara, R. R., Alain, C., and Trainor, L. J. (2006). Effects of spatial separation and stimulus probability on the event-related potentials elicited by occasional changes in sound location. Brain Res. 1071, 175-185. doi: 10.1016/j.brainres. 2005.11 .088 
Taaseh, N., Yaron, A., and Nelken, I. (2011). Stimulus-specific adaptation and deviance detection in the rat auditory cortex. PLoS One 6:e23369. doi: 10. 1371/journal.pone.0023369

Tiitinen, H., May, P., Reinikainen, K., and Näätänen, R. (1994). Attentive novelty detection in humans is governed by pre-attentive sensory memory. Nature 372, 90-92. doi: 10.1038/372090a0

Tikhonravov, D., Neuvonen, T., Pertovaara, A., Savioja, K., Ruusuvirta, T., Näätänen, R., et al. (2008). Effects of an NMDA-receptor antagonist MK-801 on an MMN-like response recorded in anesthetized rats. Brain Res. 1203, 97-102. doi: 10.1016/j.brainres.2008.02.006

Ulanovsky, N., Las, L., and Nelken, I. (2003). Processing of low-probability sounds by cortical neurons. Nat. Neurosci. 6, 391-398. doi: 10.1038/nn1032

Ulanovsky, N., Las, L., Farkas, D., and Nelken, I. (2004). Multiple time scales of adaptation in auditory cortex neurons. J. Neurosci. 24, 10440-10453. doi: 10. 1523/jneurosci.1905-04.2004

Volkov, I. O., and Galazjuk, A. V. (1991). Formation of spike response to sound tones in cat auditory cortex neurons: interaction of excitatory and inhibitory effects. Neuroscience 43, 307-321. doi: 10.1016/0306-4522(91) 90295-y

von der Behrens, W., Bäuerle, P., Kossl, M., and Gaese, B. H. (2009). Correlating stimulus-specific adaptation of cortical neurons and local field potentials in the awake rat. J. Neurosci. 29, 13837-13849. doi: 10.1523/jneurosci.3475-09. 2009

Wang, X. J., Liu, Y., Sanchez-Vives, M. V., and McCormick, D. A. (2003). Adaptation and temporal decorrelation by single neurons in the primary visual cortex. J. Neurophysiol. 89, 3279-3293. doi: 10.1152/jn.00242.2003

Wehr, M., and Zador, A. M. (2005). Synaptic mechanisms of forward suppression in rat auditory cortex. Neuron 47, 437-445. doi: 10.1016/j.neuron.2005. 06.009

Winkler, I. (2007). Interpreting the mismatch negativity. J. Psychophysiol. 21, 147163. doi: 10.1027/0269-8803.21.34.147

Winkler, I., Cowan, N., Csépe, V., Czigler, I., and Näätänen, R. (1996). Interactions between transient and long-term auditory memory as reflected by the mismatch negativity. J. Cogn. Neurosci. 8, 403-415. doi: 10.1162/jocn.1996.8. 5.403
Winkler, I., Denham, S., and Escera, C. (2013). “Auditory event related potentials," in Encyclopedia of Computational Neuroscience, eds D. Jaeger and R. Jung (Berlin Heidelberg: Springer).

Yu, X. J., Xu, X. X., He, S., and He, J. (2009). Change detection by thalamic reticular neurons. Nat. Neurosci. 12, 1165-1170. doi: 10.1038/nn.2373

Yvert, B., Crouzeix, A., Bertrand, O., Seither-Preisler, A., and Pantev, C. (2001). Multiple supratemporal sources of magnetic and electric auditory evoked middle latency components in humans. Cereb. Cortex 11, 411-423. doi: 10 1093/cercor/11.5.411

Yvert, B., Fischer, C., Bertrand, O., and Pernier, J. (2005). Localization of human supratemporal auditory areas from intracerebral auditory evoked potentials using distributed source models. Neuroimage 28, 140-153. doi: 10.1016/j. neuroimage.2005.05.056

Zhang, L. I., Tan, A. Y., Schreiner, C. E., and Merzenich, M. M. (2003). Topography and synaptic shaping of direction selectivity in primary auditory cortex. Nature 424, 201-205. doi: 10.1038/nature01796

Zhao, L., Liu, Y., Shen, L., Feng, L., and Hong, B. (2011). Stimulus-specific adaptation and its dynamics in the inferior colliculus of rat. Neuroscience 181, 163-174. doi: 10.1016/j.neuroscience.2011.01.060

Conflict of Interest Statement: The authors declare that the research was conducted in the absence of any commercial or financial relationships that could be construed as a potential conflict of interest.

Received: 17 March 2014; accepted: 24 May 2014; published online: 24 June 2014. Citation: Malmierca MS, Sanchez-Vives MV, Escera C and Bendixen A (2014) Neuronal adaptation, novelty detection and regularity encoding in audition. Front. Syst. Neurosci. 8:111. doi: 10.3389/fnsys.2014.00111

This article was submitted to the journal Frontiers in Systems Neuroscience.

Copyright (c) 2014 Malmierca, Sanchez-Vives, Escera and Bendixen. This is an openaccess article distributed under the terms of the Creative Commons Attribution License (CC BY). The use, distribution or reproduction in other forums is permitted, provided the original author(s) or licensor are credited and that the original publication in this journal is cited, in accordance with accepted academic practice. No use, distribution or reproduction is permitted which does not comply with these terms. 